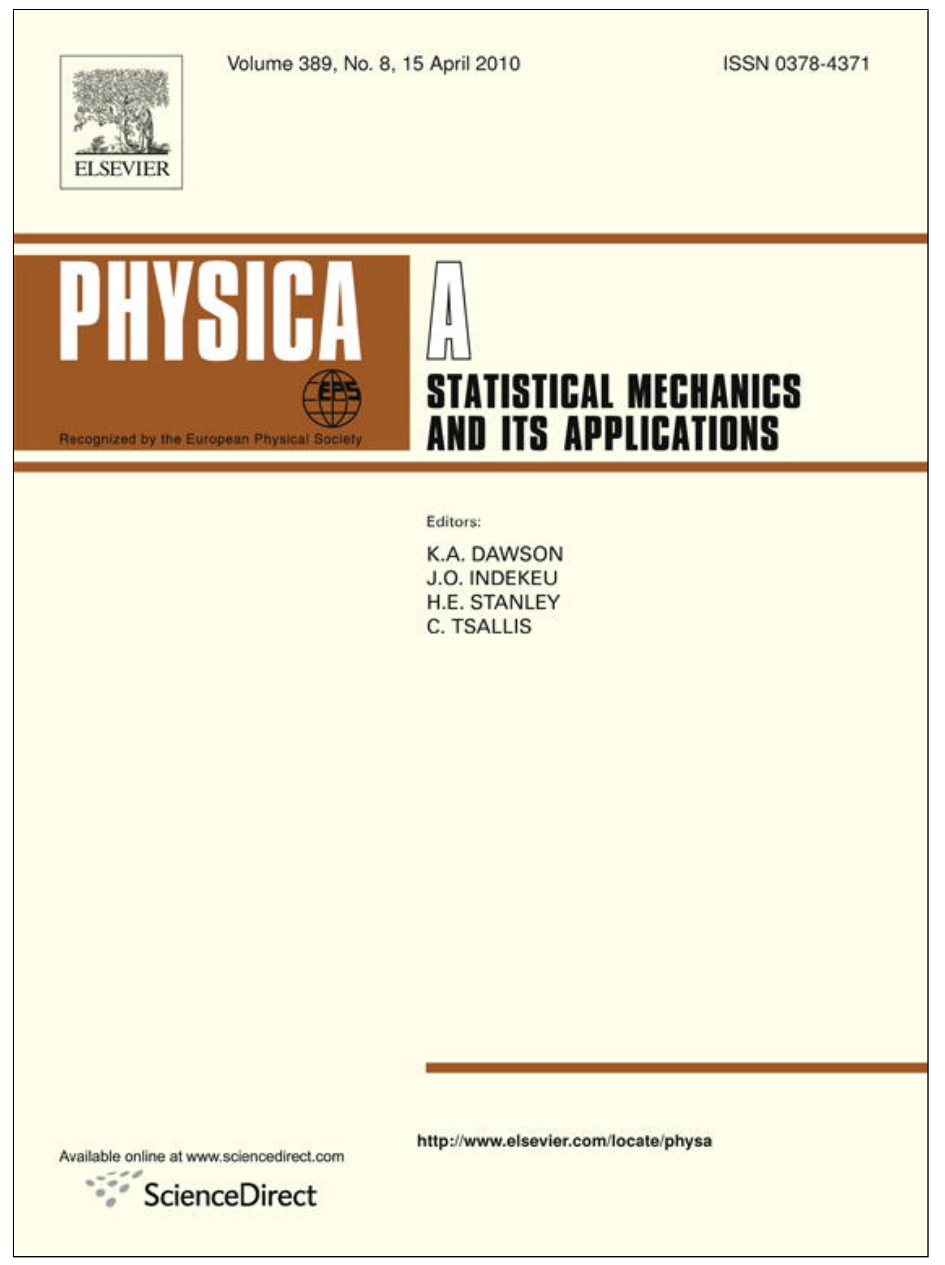

This article appeared in a journal published by Elsevier. The attached copy is furnished to the author for internal non-commercial research and education use, including for instruction at the authors institution and sharing with colleagues.

Other uses, including reproduction and distribution, or selling or licensing copies, or posting to personal, institutional or third party websites are prohibited.

In most cases authors are permitted to post their version of the article (e.g. in Word or Tex form) to their personal website or institutional repository. Authors requiring further information regarding Elsevier's archiving and manuscript policies are encouraged to visit:

http://www.elsevier.com/copyright 


\title{
Percolation of interacting particles on heterogeneous surfaces
}

\author{
M.C. Giménez, A.J. Ramirez-Pastor, F. Nieto* \\ Departamento de Física, Instituto de Física Aplicada, Universidad Nacional de San Luis - CONICET, Chacabuco 917, D5700BWS, San Luis, Argentina
}

\section{A R T I C L E I N F O}

\section{Article history:}

Received 1 August 2009

Received in revised form 25 November

2009

Available online 29 December 2009

\section{Keywords:}

Percolation

Statistical mechanics of model systems

(Ising model, Potts model, field-theory

models, Monte Carlo techniques, etc)

Monte Carlo methods

Adsorption

\begin{abstract}
A B S T R A C T
The percolation problem of interacting particles on square lattices with two kinds of energetically different sites is studied. Square lattices formed by collections of either randomly or orderly distributed sites are generated. The system is characterized by two parameters, namely, the interaction between adjacent particles, $\omega$, and the energy difference between the two kinds of sites, $\Delta E$. Particles are adsorbed at equilibrium on the lattice. By means of Monte Carlo simulations and finite-size scaling analysis the critical coverage is determined. The percolative behavior of the system is presented and discussed in terms of the mentioned parameters, $\omega$ and $\Delta E$.
\end{abstract}

(C) 2010 Elsevier B.V. All rights reserved.

\section{Introduction}

Percolation theory has attracted a great deal of interest in the past few decades and the activity in the field is still growing [1-12]. This is mainly because some aspects of the percolation process such as the geometrical phase transitions occurring in the system have gained a particular impetus due to the introduction of techniques such as Monte Carlo (MC) simulations and series expansions $[7,12]$. However, the problem is far from being exhausted.

In fact, most of the studies are devoted to the percolation of particles that are irreversibly deposited. In part this is due to the fact that the deposition (or irreversible adsorption) of particles on solid surfaces is a subject of considerable practical importance. In many experiments on adhesion of colloidal particles and proteins on solid substrates, the relaxation time scales are much longer than the times of the formation of the deposit. In such processes, the temperature of the system does not play any relevant role and it is not considered. However, in numerous systems of both theoretical and practical importance, where the adsorbed particles are in thermodynamic equilibrium, the spatial distribution of the adsorbate might be characterized by using the percolation model $[13,14]$. In previous work $[15,16]$, it has been presented a model to investigate the process of reversible adsorption of interacting particles on different lattices and studied the percolating properties of the adsorbed phase. In Refs. [15,16], the particles are always adsorbed on a homogeneous surface. This means that all the adsorption sites are considered to have the same adsorption properties as they are "seen" by the incoming particles.

By means of experimental evidence, it has been shown that even single crystal surfaces are not perfect and contain structural and energetic heterogeneities which clearly indicate the necessity to develop more refined atomistic models for describing heterogeneous surfaces and for studying the processes taken place on them as well. These models should be capable of including the characteristics of the adsorptive energy surface. The role of surface heterogeneity in many elementary processes such as adsorption, diffusion and reaction is a subject of great interest in several fields of science

\footnotetext{
* Corresponding author. Tel.: +54 2652 431080; fax: +54 2652431080.

E-mail addresses: cecigime@unsl.edu.ar (M.C. Giménez), antorami@unsl.edu.ar (A.J. Ramirez-Pastor), fnieto@unsl.edu.ar (F. Nieto).
} 
and engineering. Most materials have complex heterogeneous surfaces and all of these elementary processes are affected by surface structure.

Physical adsorption of gases has been widely used to obtain valuable information concerning to adsorptive energy distribution from experimental isotherms [17-20]. It is well known that, in addition to an adsorptive energy distribution, the adsorptive energy topography must be considered as well in order to obtain a proper characterization of surface heterogeneity. Amongst the simplest models describing surface energy topographies are the patchwise heterogeneous surface model and the independent sites model. In the former model, proposed by Ross and Olivier [21], the surface is thought as a collection of homogeneous patches, in such a way that every site within a given patch has the same adsorptive energy but different patches have different adsorption energies. In the independent sites model, after Hill [22], each adsorption site has a randomly distributed energy. In spite of simplicity, these models have allowed practical interpretations of experimental data for adsorption on a great variety of real surfaces.

In this context, it has been studied the percolation problem of interacting adsorbed particles at equilibrium on homogeneous surfaces. From this starting point, different complexities has been added to the problem in order to describe more realistic experimental situations. In Ref. [16], the influence of multisite occupancy (adsorbates occupying more than one site) has been considered. In fact, the percolation properties of an adsorbate phase of dimers was discussed. Later, the influence of surface heterogeneities on the percolation features of non-interacting particles adsorbed at equilibrium on such surfaces was presented in Ref. [23]. The present contribution goes a step further, by investigating the mutual influence of surface heterogeneities and adsorbate-adsorbate interactions on the percolation properties of the adsorbed phase. It starts by considering interacting particles adsorbed on simple heterogeneous surface in order to characterize the possible competition between these two factors. A detailed comprehension of this competition could be helpful for the evaluation of experimental studies on heterogeneous surfaces. However, it is important to note that we consider a highly idealized model here, which is not meant to reproduce a particular experimental system. Most of the problems dealing with heterogeneities attempt to characterize the surface by using a geometric parameter which gives a better idea of the behavior of surface phenomena occurring in such a surface. In this line of thinking, by knowing some of the considered parameters of the model it is possible to determine the remaining one by using as calibration curves the ones given in the paper. Thus, the model considered could help to identify the behavior of an experimentally studied system in base to the results obtained here.

This paper is organized as follows: in Section 2 we describe the lattice-gas model and the Monte Carlo simulation technique used to obtain the desired quantities for describing the percolation phase transition. Results are presented and discussed in Section 3. Finally, some conclusions are drawn in Section 4.

\section{Model and calculation method}

Let us consider the substrate is represented by a two-dimensional square lattice of $M=L \times L$ adsorption sites with periodic boundary conditions. In order to describe the system of $N$ monomers adsorbed on $M$ sites at a given temperature $T$, let us introduce the occupation variable $c_{i}$ which can take the following values:

$$
c_{i}= \begin{cases}1, & \text { if site } i \text { is occupied } \\ 0, & \text { if site } i \text { is vacant. }\end{cases}
$$

Particles can be adsorbed on the substrate with the restriction of at most one particle per site and we consider nearestneighbor $(N N)$ interaction energy $\omega$ among them. If $\varepsilon_{i}$ is the adsorption energy associated to one given surface site, the Hamiltonian of the system is given by

$$
H=\sum_{i}^{M} \varepsilon_{i} c_{i}+\omega \sum_{\langle i, j\rangle} c_{i} c_{j} .
$$

where $\langle i, j\rangle$ means summation over $N N$ pairs of sites.

Now, we describe the model used for introducing the surface heterogeneities. Let us consider the lattice-gas model introduced above as a board divided in shallow $(S)$ and deep $(D)$ sites and adsorption energies $\varepsilon_{S}$ and $\varepsilon_{D}$, respectively. We assume that the number of $S$ sites is the same as the number of $D$ sites. $S$ and $D$ sites can be put in order onto a square lattice, in such a way that any $S(D)$ site has four nearest-neighbor $D(S)$ sites, so the resulting structure will be a perfect chessboard lattice or regular patchwise lattice. But if the places in a chessboard are randomly occupied by $S$ or $D$ sites, we have a random distributed lattice. These lattices can be easily simulated on the computer.

For fixed values of surface coverage, $\theta=N / M$, adsorption energies, $\varepsilon_{S}$ and $\varepsilon_{D}$, and interaction energy between adjacent particles, $\omega$, the thermodynamic equilibrium is reached in the canonical ensemble by using a standard Kawasaki algorithm [24]. The procedure is the following. An initial arbitrary configuration of $N$ adsorbed monomers with the desired surface coverage is generated. Two sites are randomly selected, and their position are established. Then, if their occupation states are different, an attempt is made to interchange them with probability given by the Metropolis rule [25]:

$$
P=\min \left\{1, \exp \left(-\Delta H / k_{B} T\right)\right\}
$$

where $\Delta H=H_{f}-H_{i}$ is the difference between the Hamiltonians at the final and initial states and $k_{B}$ is the Boltzmann constant. 
A Monte Carlo Step (MCS) is achieved when $M$ pair of sites have been tested to exchange its occupancy state. The equilibrium state can be well reproduced after discarding the first $n^{\prime}$ MCS. Then, a set of $n$ samples in thermal equilibrium is generated by taking configurations separated from each other by a few MCS in order to avoid memory effects. We have taken $n^{\prime}=10^{3}$ and $n=2 \times 10^{4}$ for the case of ordered sites. For random site distribution, we have generated $10^{2}$ different random configurations for the surface and we have taken $n^{\prime}=10^{3}$ and $n=10^{3}$ for each case, given an average over $10^{5}$ configurations for each coverage degree.

The central idea of the percolation theory is based on finding the minimum coverage degree $\theta$ for which at least a cluster (a group of occupied sites in such a way that each one of them has at least one occupied nearest-neighbor site) extends from one side to the opposite one of the system compatible with the periodic boundary conditions present in the problem. This particular value of the coverage degree is named critical concentration or percolation threshold, $\theta_{c}$, and determines a phase transition in the system. By means of Monte Carlo simulations Hoshen, Kopelman and Monberg [26] have found the values of the effective percolation threshold with a periodic boundary conditions have been equal (within numerical errors) to the values with a free boundary condition for site (bond) process on square lattices [27-29].

As the scaling theory predicts [12], the larger the system size to study, the more accurate the values of the threshold obtained therefrom. Thus, the finite-size scaling theory give us the basis to achieve the percolation threshold and the critical exponents of a system with a reasonable accuracy. For this purpose, the probability $R=R_{L}^{X}(\theta)$ that a lattice composed of $L \times L$ sites percolates at concentration $\theta$ can be defined [2,30,31]. Here, the following definitions can be given according to the meaning of $X:$ (a) $R_{L}^{I}(\theta)=$ the probability that we find a cluster which percolates both in a rightward and in a downward direction; (b) $R_{L}^{U}(\theta)=$ the probability of finding either a rightward or a downward percolating cluster and (c) $R_{L}^{A}(\theta) \equiv \frac{1}{2}\left[R_{L}^{I}(\theta)+R_{L}^{U}(\theta)\right]$.

The first step for determining the percolation threshold consists in evaluating the effective threshold $\theta_{c}^{X}(L)$ for a lattice of finite size $L$. Once the energy difference between the two kinds of sites $\left(\Delta E \equiv \varepsilon_{S}-\varepsilon_{D}\right)$, the temperature, $T$ and the interaction energy between nearest neighbors particles $\omega$ are fixed, the next procedure is followed: (a) the construction of $n$ samples (or $10^{2} \times n$, for the case of random distribution) for a given coverage (according to the scheme mentioned before) and (b) the cluster analysis by using the Hoshen and Kopelman algorithm [32]. $n$ runs of such two steps are carried out for obtaining the number $m^{X}$ of them for which a percolating cluster of the desired criterion $X$ is found. Then, $R_{L}^{X}(\theta)=m^{X} / n$ is defined and the procedure is repeated for different values of $\theta$.

We repeat this procedure for different lattice sizes, $L$, and then we extrapolate $\theta_{c}^{X}(L)$ towards the limit $L \rightarrow \infty$ by using the finite-size scaling theory:

$$
\theta_{c}^{X}(L)=\theta_{c}(\infty)+A^{X} L^{-\frac{1}{v}}
$$

where $A^{X}$ is a non-universal constant and the critical exponent $v$ is analytically shown to be equal to $v=4 / 3$ in the case of random percolation $[2,5,7]$. The maximum of the differences between $\left|\theta_{c}^{I}(\infty)-\theta_{c}^{A}(\infty)\right|$ and $\left|\theta_{c}^{U}(\infty)-\theta_{c}^{A}(\infty)\right|$ gives the error bar for each determination of $\theta_{c}$. The interested reader is invited to see Ref. [15,30,31] for a more detailed discussion of the method for determining the critical threshold and the critical exponents.

\section{Results and discussion}

The present percolation study involves interacting adsorbed particles on heterogeneous surfaces under equilibrium conditions. In our calculations we have used $L=32,64,128$ and 160 and considered $\varepsilon_{S}=0$ without losing generality. The adsorptive energy is then characterized by the parameter $\Delta E=-\varepsilon_{D}$.

\subsection{Adsorption isotherms}

If lateral interactions are absent, the problem can be analytically solved. Let us define $\theta_{S}$ and $\theta_{D}$ as the fraction of occupied shallow and deep sites, respectively. Since the number of both types of sites is equal, the total adsorbate coverage $\theta$ is given by

$$
\theta=\frac{1}{2}\left(\theta_{S}+\theta_{D}\right)
$$

Eq. (5) does not explicitly depend on the geometrical distribution of the adsorptive sites.

The chemical potentials of adsorbed particles on the shallow and deep sites are given by

$$
\mu_{S}=\mu_{0}-\varepsilon_{S}+k_{B} T \ln \left[\frac{\theta_{S}}{1-\theta_{S}}\right]
$$

and

$$
\mu_{D}=\mu_{o}-\varepsilon_{D}+k_{B} T \ln \left[\frac{\theta_{D}}{1-\theta_{D}}\right] .
$$



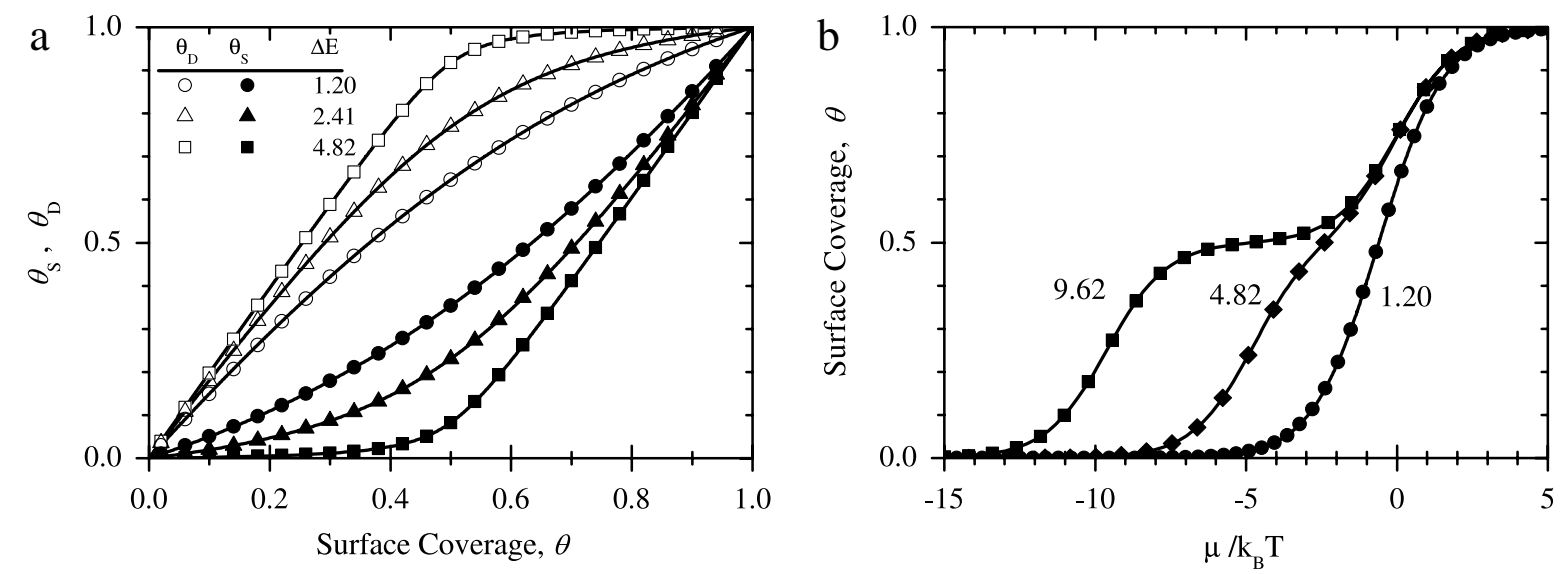

Fig. 1. (a) Site specific coverages, $\theta_{S}$ (filled symbols) and $\theta_{D}$ (empty symbols) versus total coverage, $\theta$ and (b) Adsorption isotherms, $\theta$ vs $\mu / k_{B} T$. Results are shown for three characteristic temperatures, expressed in terms of $\left(\varepsilon_{S}-\varepsilon_{D}\right) / k_{B} T$, as indicated. Symbols denote Monte Carlo results, while solid lines represent thermodynamical calculations according to Eqs. (5) and (8). The figure shows the case when no ad-ad interactions are present.
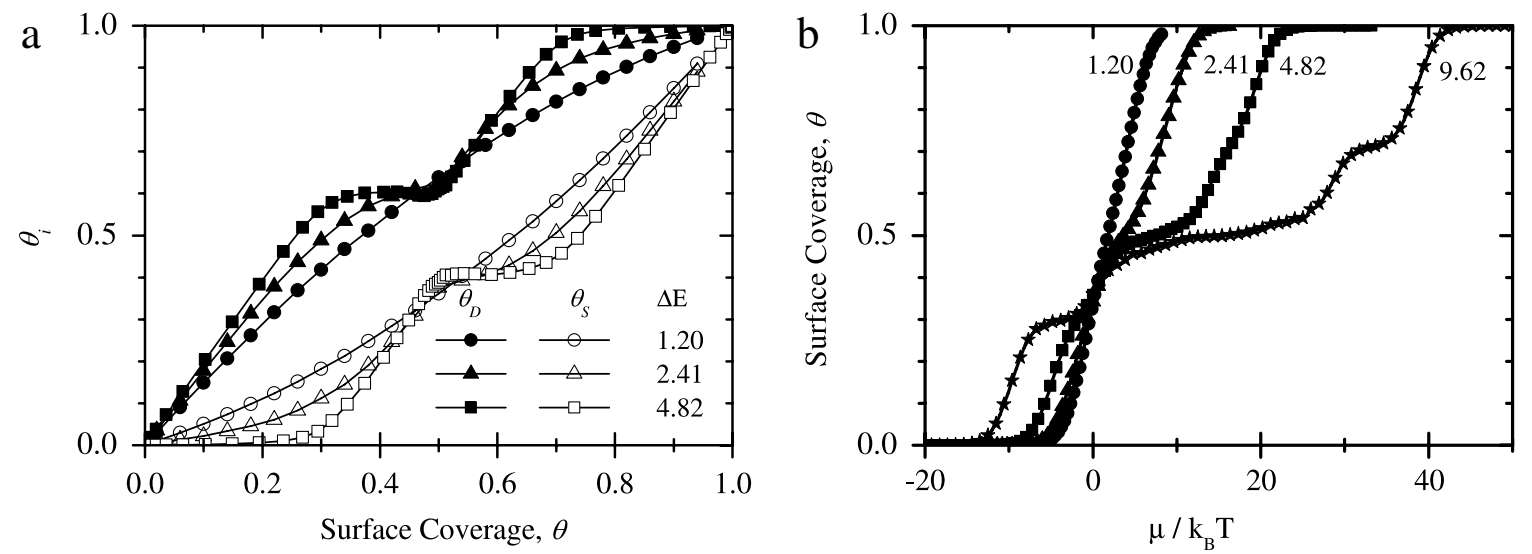

Fig. 2. (a) Site specific coverage, $\theta_{S}$ (filled symbols), $\theta_{D}$ (empty symbols) versus total coverage $\theta$ and (b) adsorption isotherms, $\theta$ vs $\mu / k_{B} T$ for three characteristic temperatures, expressed in terms of $\left(\varepsilon_{S}-\varepsilon_{D}\right) / k_{B} T$ as indicated. The figure shows the case when repulsive ad-ad interactions are also present.

Here $\mu_{o}$ is the chemical potential of an ideal Langmuir gas. In the case of thermodynamical equilibrium we have $\mu_{S}=\mu_{D}$, and thus

$$
\ln \left[\frac{\theta_{D}\left(1-\theta_{S}\right)}{\theta_{S}\left(1-\theta_{D}\right)}\right]=\left(\frac{\varepsilon_{D}-\varepsilon_{S}}{k_{B} T}\right) .
$$

It is important to note that Eqs. (5)-(8) do not depend on the surface topography, i.e. on the distribution of deep and shallow sites.

Solving Eqs. (5) and (8) we obtain the coverage dependencies of the site specific surface coverages, $\theta_{S}(\theta)$ and $\theta_{D}(\theta)$; see Fig. 1a. As is intuitively expected, for a given total coverage $\theta$, the partial coverage of deep sites, $\theta_{D}$, is always larger than the partial coverage of shallows sites, $\theta_{S}$; and is also evident that the effect of surface heterogeneities is more important at high values of $\Delta E / k_{B} T$.

Solving Eqs. (5)-(7) allows the calculation of adsorption isotherms such as shown in Fig. 1b. At high $\Delta E / k_{B} T$ the sequential occupation of deep and shallow sites is observed, and a step occurs after saturation of the deep sites.

Now, it is interesting to know how the adatoms are distributed over the deep and shallow sites when lateral interactions are present in the system.

For this case we have to distinguish two very different behaviors according to whether the heterogeneities in the system are ordered or randomly distributed. In the former case, the qualitative behavior is quite similar to the non-interacting case. The incoming particles prefer to be adsorbed in one of the two interpenetrated lattices of the system (formed by the deep sites) and an ordered phase is formed. Upon increasing the ad-ad repulsive interactions and/or the interaction energy between $N N$ particles the preference for the deep-site lattice is enhanced. The plots of the partial coverages $\theta_{S}$ and $\theta_{D}$ and the adsorption isotherms are quite similar to those shown in Fig. 1.

A more interesting case is when the deep sites are randomly distributed. In Fig. 2a $\theta_{S}(\theta)$ and $\theta_{D}(\theta)$ are shown for three representative values of the ratio $\Delta E / k_{B} T$. In the figure, all calculations are performed assuming $\Delta E / k_{B} T=|\omega| / k_{B} T$, i.e. the energy difference between both types of sites is numerically equal to the absolute value of the nearest-neighbor repulsion. 

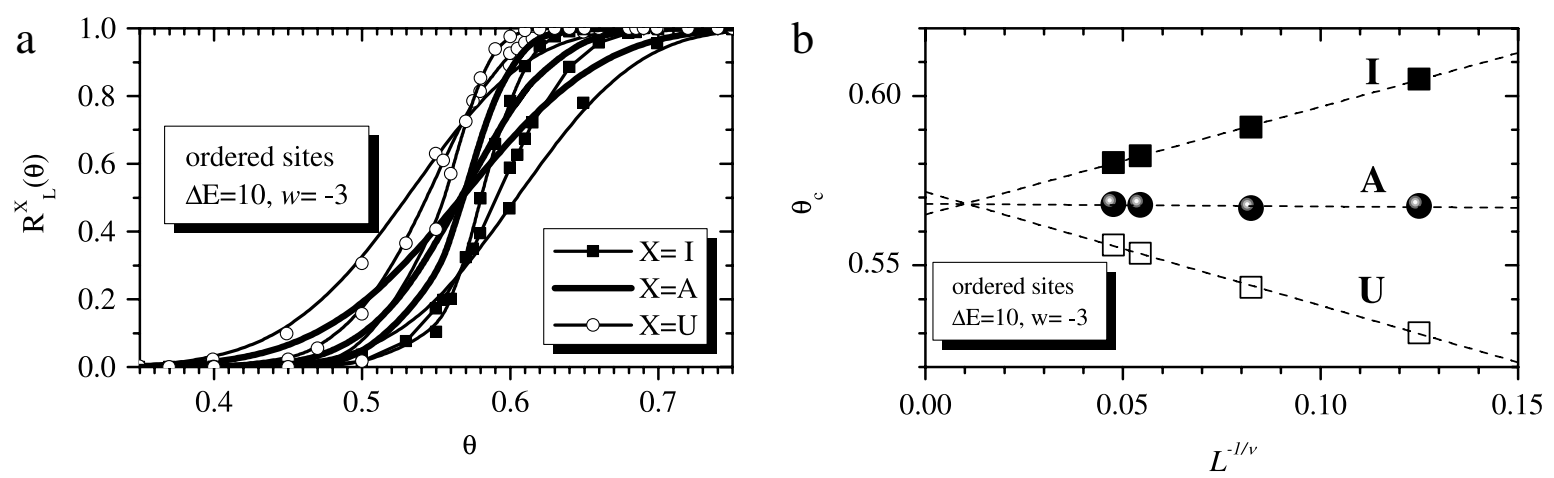

Fig. 3. (a) Percolation probability as a function of the coverage degree for several lattice sizes $(L=32, L=64$ and $L=160)$ and the three criteria ( $X=I$, $X=A$ and $X=U)$. (b) Percolation threshold as a function of the lattice size for the three criteria $(X=I, X=A$ and $X=U)$. The intersection of the lines with the $y$-axis represents the extrapolation for $L=\infty$. We have taken the case of ordered sites with $\Delta E=10$ and $\omega=-3$ as an example.

At the lowest value of $\Delta E / k_{B} T$ considered in this work, $\Delta E / k_{B} T=1.20$ the curves are very similar with respect to the corresponding ones of the non-interacting case. At such temperature $\theta_{D}$ is always larger than $\theta_{S}$, indicating the preferential occupation of deep sites even if repulsive interactions are present.

Upon increasing $\Delta E / k_{B} T$, the influence of the heterogeneity becomes more pronounced and the differences between $\theta_{D}$ and $\theta_{S}$ are enhanced. This is intuitively expected. However, it is quite interesting to realize that, if $\theta$ approaches half coverage, (i) $\theta_{D}$ goes through a relative minimum and (ii) $\theta_{S}$ goes through a relative maximum. In order to explain these peculiarities we note that the $N N$ repulsive interactions induce long-range $c(2 \times 2)$ ordering around half coverage. We conclude that in a small range of coverages around $\theta \approx 0.5$ the behavior of $\theta_{S}$ and $\theta_{D}$ is dominated by the $N N$ repulsive interactions and the $c(2 \times 2)$ ordering. However, for total coverages far away from half coverage the behavior is largely controlled by the surface heterogeneity.

These ideas are reinforced by studies of adsorption isotherms (Fig. 2b) which were obtained by using the Monte Carlo algorithm in the grand-canonical ensemble. The figure shows the adsorption isotherms for several representative values of the ratio $\Delta E / k_{B} T$. There are strong deviations from Langmuir behavior (non-interacting lattice gas on a homogeneous surface) especially at low temperature where the influence of surface heterogeneities and ad-ad interactions becomes important. The corresponding adsorption isotherms exhibit characteristic plateaus, which are most pronounced at highest values of $\Delta E / k_{B} T$. The first plateau is considered as a consequence of the preferential adsorption of deep sites. As the concentration of the deep sites is equal to 0.5 , the probability for a deep site to be located in the direct vicinity of another deep site is quite large. Representative snapshots of lattice-gas configurations show clusters of adjacent deep sites on the surface. Thus, the effect of the $N N$ repulsive interactions prevents the occupation of deep sites in such clusters (causing frustration) and, as a consequence, the first step is established at $\theta<0.5$. After this first plateau, the fraction of adatoms on shallow sites increases. Close to half coverage $\theta_{D}$ is significantly reduced due to the formation of a long-range ordered $c(2 \times 2)$ structure, and this corresponds to the second plateau of the adsorption isotherm. Sharply above half coverage the empty deep sites are occupied, and this corresponds to the third plateau of the isotherms. Upon further increasing the chemical potential the saturation of the shallow sites occurs, and this corresponds to the final plateau; see Fig. $2 \mathrm{~b}$.

\subsection{Finite-size scaling treatment}

As it was explained above, for a given value of $L, \omega / k_{B} T$ and $\Delta E / k_{B} T$, a curve of $R_{L}^{X}$ as a function of $\theta$ is obtained for the three different criteria $X=A, X=I$ and $X=U$. Fig. 3a shows the curves for the three mentioned criteria and three system sizes $(L)$ for the case of $\Delta E / k_{B} T=10$ and $\omega / k_{B} T=-3$. As $L$ increases, the transition between $R_{L}^{X}=0$ and $R_{L}^{X}=1$, becomes more abrupt and the limit case (for $L=\infty$ ) would be a step function with a well-defined $\theta_{c}$ (with $R^{X}=0$ for $\theta<\theta_{c}$ and $R^{X}=1$ for $\theta>\theta_{c}$ ).

In order to find the percolation threshold $\theta_{c}$ for $L=\infty$, it is necessary to make an extrapolation based on Eq. (4). Fig. 3b shows the curves of $\theta_{c}$ as a function of $L^{-1 / v}$ for the three criteria $(X=A, I$ and $U)$ in the considered example. The value of the exponent $v$ is calculated also by finite-size scaling for each case (see Refs. [15,16,23,30,31] for more details). The intersection of the lines with the $y$-axes represents the value of $\theta_{c}$ for $L=\infty$. The difference of the extrapolated values employing the three criteria gives an estimation of the uncertainty in the calculated percolation threshold.

\subsection{Phase diagrams}

The percolation threshold, $\theta_{c}$, is plotted in Figs. 4 and 5 (6 and 7) as a function of $\Delta E / k_{B} T$ and $\omega / k_{B} T$ for the case of ordered (random) distribution of deep sites, respectively. Each curve represents a phase diagram separating a percolating from a non-percolating region.

Fig. 4 shows $\theta_{c}$ as a function of $\Delta E / k_{B} T$ for four values of $\omega / k_{B} T$ in the case of ordered sites. The case with $\omega / k_{B} T=0$ corresponds to non-interacting particles and has been studied in previous work [23]. 


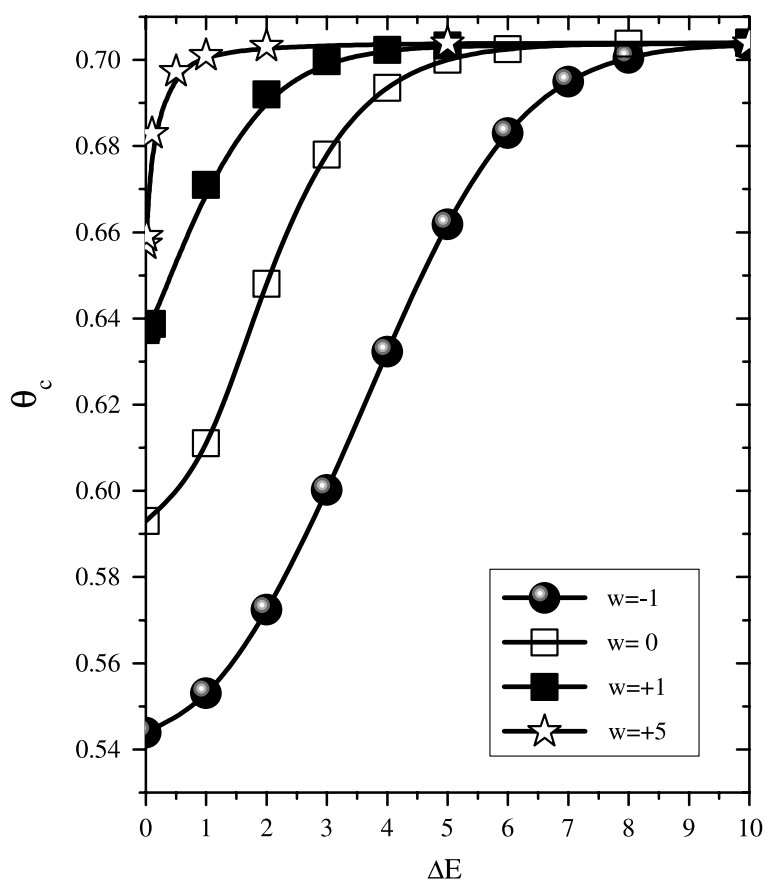

Fig. 4. Percolation threshold $\left(\theta_{c}\right)$ as a function of $\Delta E / k_{B} T$ for several values of $\omega / k_{B} T$ in the case of ordered sites.

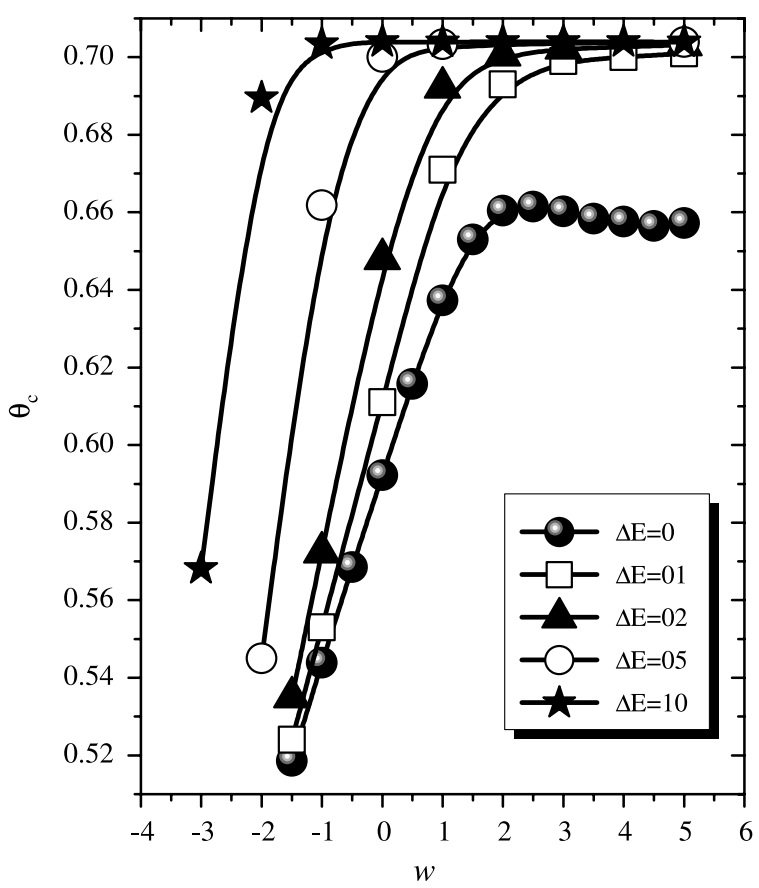

Fig. 5. Percolation threshold $\left(\theta_{c}\right)$ as a function of $\omega / k_{B} T$ for several values of $\Delta E / k_{B} T$, in the case of ordered sites.

It is possible to observe that attractive interactions $\left(\omega / k_{B} T=-1\right)$ between particles result in a curve below the non-interacting case $\left(\omega / k_{B} T=0\right)$. That means that the percolation threshold is minor and the system percolates more easily. It can be understood by taking into account that attractive interactions produce a more compact structure and favor coalescence of particles and clusters.

On the other hand, repulsive interactions result in an increment of the percolation threshold. We can notice the limiting value $\theta_{c} \approx 0.7$. This value can be understood in terms of effective neighbors (see Refs. [23,33]). A brief explanation would be that for $\theta=0.5$, a perfect chessboard structure is obtained. When new particles are added, they can be seen as disposed in an effective lattice in which each empty site has eight effective $N N$. The percolation threshold for a lattice with this coordination number (a square lattice with connections between nearest and next-nearest neighbors) is $\theta=0.41$ [5]. As these sites represent a half of the whole lattice, this coverage added to the number of particles already adsorbed in the chessboard $(\theta=0.5)$, gives the observed saturation value of $\theta=0.7$. A larger value of $\omega / k_{B} T$ together with a high $\Delta E / k_{B} T$ enhance the described effect. 


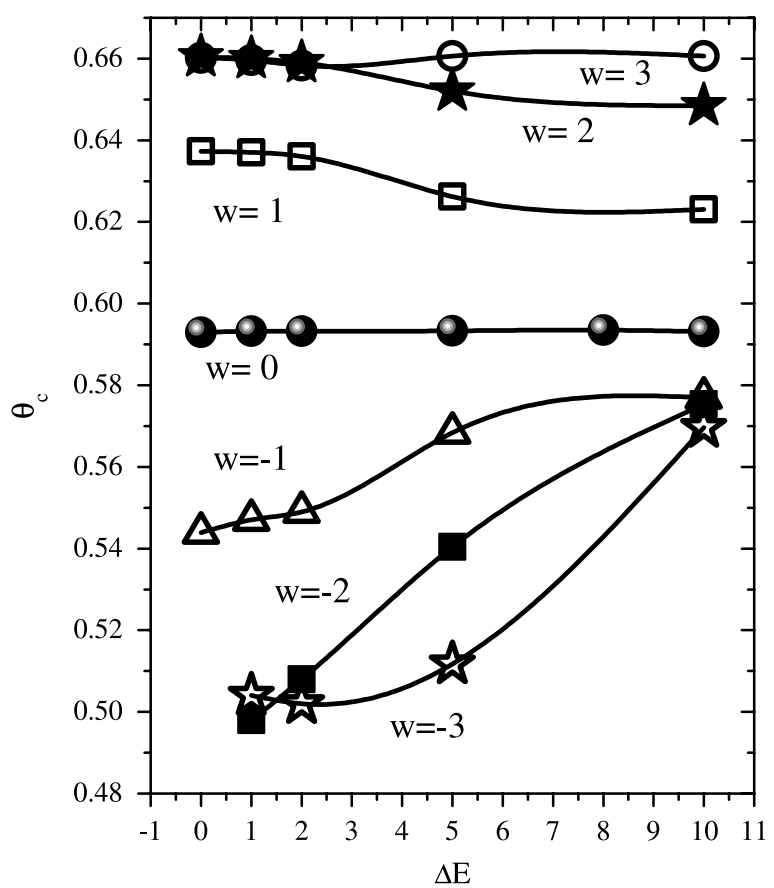

Fig. 6. Percolation threshold $\left(\theta_{c}\right)$ as a function of $\Delta E / k_{B} T$ for several values of $\omega / k_{B} T$ in the case of random distribution of sites.

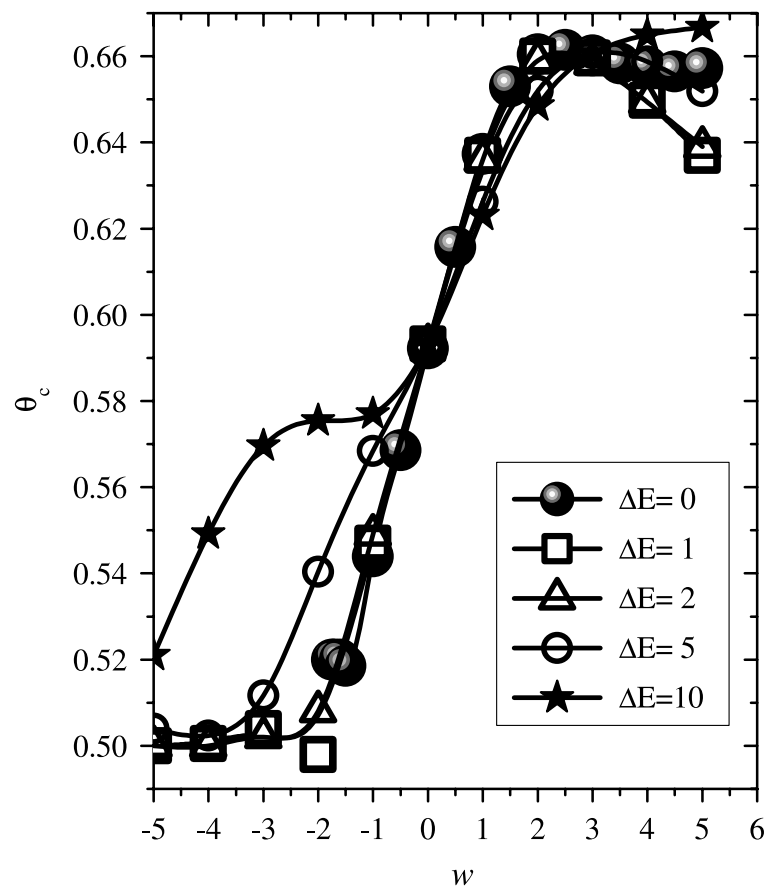

Fig. 7. Percolation threshold $\left(\theta_{c}\right)$ as a function of $\omega / k_{B} T$ for several values of $\Delta E / k_{B} T$ in the case of random distribution of sites.

Fig. 5 shows $\theta_{c}$ as a function of $\omega / k_{B} T$ for five values of $\Delta E / k_{B} T$ in the case of ordered sites. The case with $\Delta E / k_{B} T=0$ corresponds to a homogeneous surface and has been studied in previous work $[15,16]$. One can see that for each value of $\Delta E / k_{B} T, \theta_{c}$ increases with the increment of $\omega / k_{B} T$ (increasing repulsion between particles), until the limiting value of $\theta_{c} \approx 0.7$ (except for $\Delta E / k_{B} T=0$ ), as it has been analyzed before. It means that even for small values of $\Delta E / k_{B} T$, the repulsion between particles produces the chessboard structure and the effect of effective neighbors can be observed. We also can notice that upon increasing $\Delta E / k_{B} T$ the critical curve $\theta_{c}\left(\Delta E / k_{B} T\right)$ is raised.

Fig. 6 shows $\theta_{c}$ as a function of $\Delta E / k_{B} T$ for seven values of $\omega / k_{B} T$ in the case of random distribution of deep sites. The case of $\omega / k_{B} T=0$ corresponds to non-interacting particles and $\theta_{c}$ is independent of $\Delta E / k_{B} T$. This particular case has been discussed in previous work [23]. Like in the ordered case, attractive values of $\omega / k_{B} T$ produce minor values of $\theta_{c}$ and vice versa. In general, for each value of $\omega / k_{B} T, \theta_{c}$ does not depend very much on $\Delta E / k_{B} T$. Nevertheless, there is a general tendency: as $\Delta E / k_{B} T$ increases, $\theta_{c}$ approaches to the non-interacting case. This can be understood by considering 
a

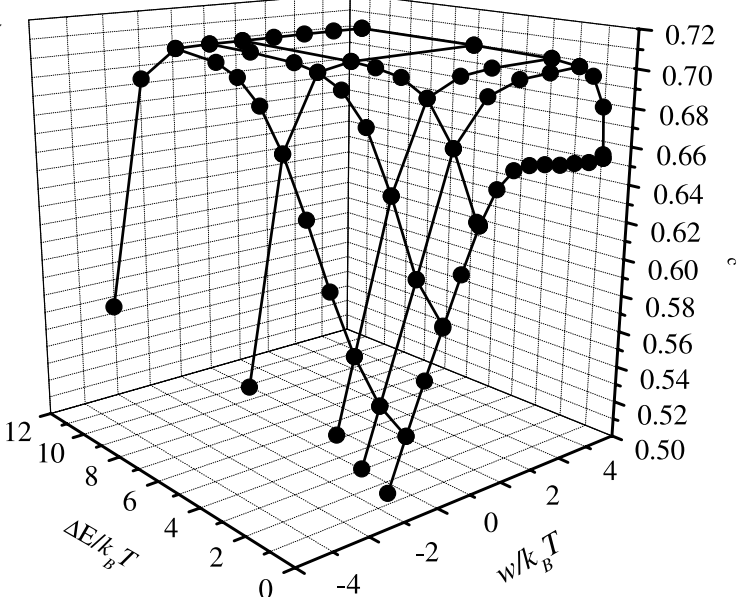

b

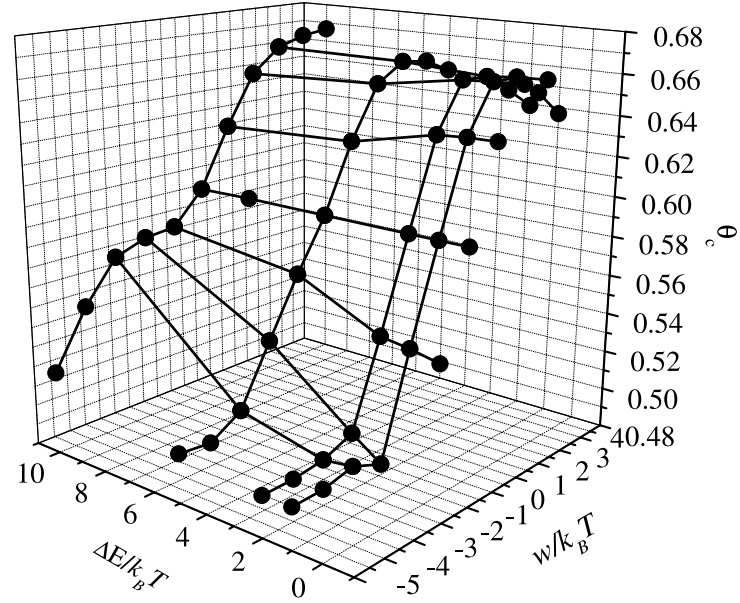

Fig. 8. Three-dimensional plots where $\Delta E / k_{B} T, \omega / k_{B} T$ and $\theta_{c}$ are considered in the axes, for (a) ordered and (b) disordered lattices, respectively. The surface shown in the figures divide the percolation from the non-percolation regions according to the values of the parameters.

that random distribution of deep sites, together with high energy differences between sites, favors a random distribution of particles and lowers the effect of the lateral interaction between them.

Fig. 7 shows $\theta_{c}$ as a function of $\omega / k_{B} T$ for five values of $\Delta E / k_{B} T$ in the case of random distribution of deep sites. Again, the case with $\Delta E / k_{B} T=0$ corresponds to an homogeneous surface $[15,16]$. For small values of $\Delta E / k_{B} T$, the curves are very similar to the homogeneous case. Only for values of $\Delta E / k_{B} T=5$ or 10 we can appreciate a departure of the curves especially for negative values of $\omega / k_{B} T$ (attractive interactions). This situation can be understood considering that random distribution of deep sites with great energy differences alters the coalescence of the compact islands and increases the value of $\theta_{c}$.

The complete scenario is a three-dimensional plot where $\Delta E / k_{B} T, \omega / k_{B} T$ and $\theta_{c}$ are considered. Fig. $8 \mathrm{a}$ and b show these three-dimensional graphics for ordered and disordered lattices, respectively. The surface shown in the figures divide the percolation from the non-percolation regions according to the values of the parameters.

\section{Conclusions}

In the present paper we have studied the percolation behavior of the adsorbed monolayer composed by interacting particles on a simple heterogeneous surface. In the framework of this model, the surface was assumed to be formed by two kinds of different adsorption sites. Each kind of adsorption site is characterized by an adsorption energy, $\varepsilon_{S}$ or $\varepsilon_{D}$. We call them shallow and deep sites, respectively, which are (i) arranged in a chessboard-like ordered structure or (ii) randomly located. For simplicity we assume that the number of deep adsorption sites is equal to the number of shallow adsorption sites.

We have used the Monte Carlo technique in order to determine the equilibrium conditions of the system. The thermodynamic equilibrium is reached in the canonical ensemble by using a standard Kawasaki algorithm [24]. Once the equilibrium is established, then we consider each independent state in order to analyze the percolation transition. The numerical scheme used for that is based on the Hoshen and Kopelman algorithm [32]. Finally, the determination of $\theta_{c}(\Delta E, \omega)$ is done by using extensively the finite-size scaling analysis.

We conclude this work remarking the distinctive qualities of the site percolation problem on simple heterogeneous lattices. $\theta_{c}(\Delta E)$ for fixed values of $\omega / k_{B} T$ shows a different behavior according the heterogeneity in the surface is ordered or randomly generated. In the former case, $\theta_{c}(\Delta E)$ slowly grows up to a saturation value as the energy difference between shallow and deep patches increases. The limiting value, $\theta_{c} \approx 0.7$ can be understood in terms of effective neighbors. A larger value of $\omega / k_{B} T$ together with a high $\Delta E / k_{B} T$ enhance the described effect. For attractive interactions, $\theta_{c}(\Delta E)$ results in a curve below the non-interacting case $\left(\omega / k_{B} T=0\right)$ which means that the percolation threshold is minor. Thus, attractive interactions produce a more compact structure and the system percolates more easily. For a random distribution of deep sites, attractive values of $\omega / k_{B} T$ produce minor values of $\theta_{c}$. For each value of $\omega / k_{B} T, \theta_{c}$ does not depend very much on $\Delta E / k_{B} T$. However, a general tendency can be drawn: as $\Delta E / k_{B} T$ increases, $\theta_{c}$ approaches to the non-interacting case. This can be understood by considering that random distribution of deep sites, together with high energy differences between sites, favors a random distribution of particles and lowers the effect of lateral interaction between them.

$\theta_{c}(\omega)$ increases with the increment of $\omega / k_{B} T$ until the limiting value of $\theta_{c} \approx 0.7$ for ordered distribution of deep sites. Even for small values of $\Delta E / k_{B} T$, the repulsion between particles produces the chessboard structure and the effect of effective neighbors can be observed. For random distribution of deep sites, it is important to emphasize that a high value of $\Delta E / k_{B} T$ alters the coalescence of the compact islands and increases the value of $\theta_{c}$.

\section{Acknowledgements}

This work was supported in part by CONICET (Argentina) under project PIP 112-200801-01332; Universidad Nacional de San Luis (Argentina) under project 322000 and the National Agency of Scientific and Technological Promotion (Argentina) 
under project 33328 PICT 2005. The numerical work were done using the BACO parallel cluster (composed by 60 PCs each with a 3.0 GHz Pentium-4 processor and 90 PCs each with a $2.4 \mathrm{GHz}$ Core 2 Quad processor) located at Instituto de Física Aplicada, Universidad Nacional de San Luis - CONICET, San Luis, Argentina.

\section{References}

[1] J.M. Hammersley, Proc. Camb. Phil. Soc. 53 (1957) 642.

[2] D. Stauffer, Introduction to Percolation Theory, Taylor \& Francis, 1994.

[3] G. Deutscher, R. Zallen, J. Adler (Eds.), Percolation Structures and Processes, in: Annals of the Israel Physical Society, vol. 5, Ayalon Offset Ldt, 1983.

[4] M. Sahimi, Application of the Percolation Theory, Taylor \& Francis, London, 1992.

[5] R. Zallen, The Physics of Amorphous Solids, John Willey \& Sons, NY, 1983.

[6] S. Kirkpatrick, Rev. Modern Phys. 45 (1973) 574

[7] J.W. Essam, Rep. Progr. Phys. 43 (1980) 843.

[8] J.-P. Hovi, A. Aharony, Phys. Rev. B 53 (1996) 235.

[9] G. Deutscher, R. Zallen, J. Adler (Eds.), Percolation Structures and Processes, in: Annals of the Israel Physical Society, vol. 5, Adam Hilger, Bristol, 1983.

[10] V. Cornette, A.J. Ramirez-Pastor, F. Nieto, Physica A 327 (2003) 71.

[11] V. Cornette, A.J. Ramirez-Pastor, F. Nieto, European J. Phys. B 36 (2003) 391.

[12] K. Binder, Rep. Progr. Phys. 60 (1997) 488.

[13] Z. Gao, Z.R. Yang, Physica A 255 (1998) 242.

[14] K.Y. Gao, B.X. Liu, J. Phys.: Condens. Matter 11 (1999) 39.

[15] M.C. Giménez, F. Nieto, A.J. Ramirez-Pastor, J. Phys. A Math. Gen. 38 (2005) 1-12.

[16] M.C. Giménez, F. Nieto, A.J. Ramirez-Pastor, J. Chem. Phys. 125 (2006) 184707.

[17] W.A. Steele, The Interaction of Gases with Solid Surface, Pergamon Press, New York, 1974.

[18] M. Jaroniec, P. Bräuer, Surf. Sci. Rep. 6 (1986) 65.

[19] M. Jaroniec, R. Madey, Physical Adsorption on Heterogeneous Solids, Elsevier, Amsterdam, 1988.

[20] W. Rudzinski, D.H. Everett, Adsorption of Gases on Heterogeneous Surfaces, Academic Press, New York, 1992.

[21] S. Ross, J.P. Olivier, On Physical Adsorption, Willey \& Sons, New York, 1964.

[22] T.L. Hill, J. Chem. Phys. 17 (1949) 520.

[23] M.C. Giménez, A.J. Ramirez-Pastor, F. Nieto, Physica A 387 (2008) 6526.

[24] K. Kawasaki, C. Domb, M. Green (Eds.), Phase Transitions and Critical Phenomena, vol. 2, Academic, London, 1972.

[25] N. Metropolis, A.W Rosenbluth, M.N. Rosenbluth, A.H. Teller, E. Teller, J. Chem. Phys. 21 (1953) 1087.

[26] J. Hoshen, R. Kopelmann, E.M. Monberg, J. Stat. Phys. 19 (1978) 219.

[27] D.W. Herrmann, D. Stauffer, Z. Phys. B 40 (1980) 133.

[28] R.M. Ziff, Phys. Rev. Lett. 69 (1992) 2670.

[29] M.S. Watanabe, Phys. Rev. E 51 (1995) 3945

[30] F. Yonezawa, S. Sakamoto, M. Hori, Phys. Rev. B 40 (1989) 636

[31] F. Yonezawa, S. Sakamoto, M. Hori, Phys. Rev. B 40 (1989) 650.

[32] J. Hoshen, R. Kopelman, Phys. Rev. B 14 (1976) 3428.

[33] F. Nieto, A.J. Ramirez Cuesta, R.J. Faccio, Phys. Rev. E 59 (1999) 3706. 